\title{
Does cage quantum delocalisation influence the Translation-Rotational bound states of molecular hydrogen in clathrate hydrate?
}

David M. Benoit, ${ }^{1,}$ ) David Lauvergnat, ${ }^{2}$ and Yohann Scribano ${ }^{3}$

1) E.A. Milne Centre for Astrophysics $\&$ G.W. Gray Centre for Advanced Materials, Chemistry, The University of Hull, Cottingham Road, Kingston upon Hull HU6 7RX, $U K$

2) Laboratoire de Chimie Physique, CNRS, Univ. Paris-Sud, Université Paris-Saclay, 91405, Orsay, France

3) Laboratoire Univers et Particules de Montpellier, Université de Montpellier, LUPM - UMR CNRS 5299, 34095 Montpellier Cedex, France

(Dated: 14 May 2018) 
In this study, we examine the effect of a flexible description of the clathrate hydrate framework on the translation-rotation (TR) eigenstates of guest molecules such as molecular hydrogen. Traditionally, the water cage structure is assumed to be rigid, thus ignoring the quantum nature of hydrogen nuclei in the water framework. However, it has been shown that protons in a water molecule possess a marked delocalised character in many situations, ranging from water clusters to proton transfer in the bulk. In the case of water clathrates, all previous TR bound-state calculations of guest molecules consider that the caging water molecules are fixed at their equilibrium geometry. Only recently, a static investigation of the role of proton configurations was performed by Bačić and co-workers by sampling a very large number of different static structures of water clathrates.

Here, we investigate the importance of the rotational degrees of freedom of the water cage on the TR levels of guest molecule using an efficient adiabatic decoupling scheme. Our approach combines rigid body Diffusion Monte Carlo calculations for the description of the rotational degree of freedom of water molecules surrounding the guest molecular hydrogen to an efficient Smolyak sparse-grid technique for the calculation of the TR levels. This approach allows us to take into account the highly anharmonic nature of the rotational water motions in a high-dimensional system. The clathrate-induced splittings of the $j=1$ rotational levels are much more sensitive to the quantum hydrogen delocalisation than the translational transitions. This results is in good agreement with the previous static study of Bačić and co-workers.

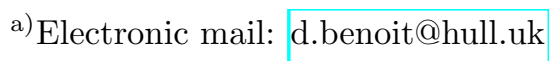




\section{INTRODUCTION}

Clathrate hydrates are a type of inclusion compounds that are formed when water and guest molecules come into contact to create an ice-like framework of hydrogen bonds. The dynamics of molecular hydrogen encapsulated in clathrates hydrates has been investigated both experimentally and theoretically for several decades due to their fundamental interest and also for their potential as hydrogen storage materials ${ }^{1}$. The confinement of hydrogen in water cages results in the quantisation of the translational degrees of freedom associated to its center of mass displacement of the hydrogen molecule and their coupling to its quantised rotational motions. These structures have been studied using a number of experimental spectroscopy techniques, such as inelastic neutron spectroscopy (INS) and Raman spectroscopy. Most research efforts have focused on a small structure, the sII clathrate hydrate (there are three known clathrate hydrate structures: sI, sII, and $\mathrm{sH}$ ), which was

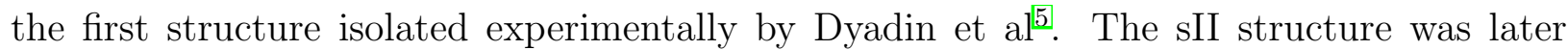
extensively studied by Mao et al. ${ }^{677}$ who demonstrated that molecular hydrogen does form clathrate hydrates. Those experiments are usually conducted at low temperatures where the confined hydrogen molecule in this nanocage becomes a highly quantum mechanical system due to its small size, mass and large rotational constant.

In order to help assignment of the measured INS spectra and to perform a quantitative analysis of such inclusion compound system, several theoretical studies were previously done to calculate the translational-rotation (TR) levels and make a comparison with experimental data. Indeed such calculations represent a very sensitive test of the quality of the potential energy surface (PES) used in each simulation. This is of crucial importance since the ultimate goal for theoreticians is to develop an accurate PES with reliable predictive ability. The research field of encapsulated hydrogen in clathrate hydrates has greatly benefited from the contributions of Bačić and coworkers, who have made rigorous calculations of a rigid hydrogen molecule in a clathrate hydrate structure over the years ${ }^{23}$. In those calculations, the cage is mostly considered as rigid (along with the hydrogen molecule) and the interaction between the hydrogen molecule and the water cage is described using a sum of pairwise dimer potential $\mathrm{H}_{2}-\mathrm{H}_{2} \mathrm{O}$. They used both empirical and ab initio pair potentials to compute the TR levels and compared those to experimental data. The best agreement with the experimental data thus far was obtained using the empirical simple point charge model SPC/E. The most 
recent calculations, using an ab initio potential computed with pairwise water-hydrogen dimer potential, V08 ${ }^{24}$, have shown to overestimate angular anisotropy ${ }^{25}$.

One interest of those theoretical studies is to use spectroscopy as a probe of the possible multiple occupancy of hydrogen in those clathrate structure. Therefore, fully quantum calculations of the ground state were performed at $T=0 \mathrm{~K}$ using Quantum Diffusion

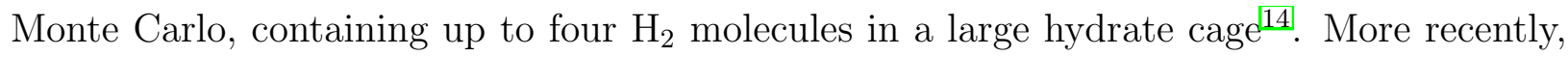
TR eigenstates have also been obtained for $\mathrm{two}^{\underline{26127}}$ and four ${ }^{\underline{28}} \mathrm{H}_{2}$ molecules inside a large clathrate hydrate cage. The effect of the temperature was also investigated using pathintegral molecular dynamics (PIMD) simulations $\frac{16}{16}$. However, those studies have considered a rigid structure of the hydrogen-bonded framework. Plattner and Meuwly ${ }^{29}$ have investigated quantum effects on the $\mathrm{H}_{2}$ vibrational frequency shifts in clathrate hydrates by combining classical molecular dynamics (MD) and path integral MD simulations (even for the water molecules) with electronic structure calculations at the DFT (B3LYP) and MP2 levels, for a system of $2 \times 2 \times 2$ unit cells. Using vibrational frequency analysis, they concluded that $\mathrm{H}_{2}$ is sensitive to the environment and to quantum effects. Powers et al. have also investigated the effect of the orientation of each water molecule within a condensed environment on the TR levels 20 . They have considered several water domains of increasing size encapsulating a single $\mathrm{H}_{2}$ molecule. For each domain size, several hundred distinct hydrogen-bonding topologies were considered in order to simulate the effects of proton disorder in a static way. This study revealed that the $j=1$ rotational splitting is increased significantly by the interactions of $\mathrm{H}_{2}$ with the water molecules beyond the central small cage, and depends strongly on the distribution of the water protons. However, the splitting of the translational fundamental levels changes very little with the inclusion of the condensed-phase water and is only weakly sensitive to proton disorder.

In this paper, we investigate the influence of quantum delocalisation of the water environment (at least in the first shell surrounding the molecular hydrogen) on the TR bound states. We use a combination of two different tools to tackle this very challenging system with many degrees of freedom, namely rigid body Diffusion Monte Carlo calculations for the description of the rotational degree of freedom of water molecules surrounding the guest molecular hydrogen and an efficient Smolyak sparse-grid technique for the calculation of the TR levels.

The paper is organised as follows: our methodology is described in Section II. In Section 
III, we present and discuss the results. Section IV summarises the work and outlines possible directions for future research.

\section{METHODOLOGY}

\section{A. Clathrate cage structures}

In order to account for size effects, we define three spherical sII clathrate hydrate domains of increasing radius. Each of those contains an increasing number of $\mathrm{H}_{2} \mathrm{O}$ molecules around the small dodecahedral cage at the center, following the approach of Bačić and collaborators ${ }^{20}$. The structures of water domains are the same as the ones used in the recent study of Powers et al ${ }^{25}$. The $\left(\mathrm{H}_{2} \mathrm{O}\right)_{n}$ structures were generated using (a) a spherical domain with a cutoff radius of $5.0 \AA$ leading to a $n=20$ water molecule cage, the dodecahedral clathrate cage itself. (b) A domain obtained with an increased cutoff radius of $7.5 \AA$, leading to a two-layer system of $n=40$ water molecules. (c) Finally a structure obtained using a cutoff radius of $9.0 \AA$, adding a further shell of 36 water molecules, to produce a system containing $n=76$ water molecules. The structures of the three domains (a)-(c) are shown in Fig. 1 below without guest molecule.

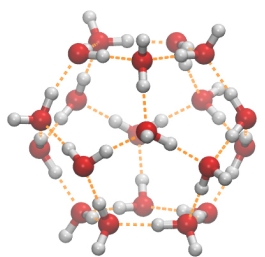

(a)

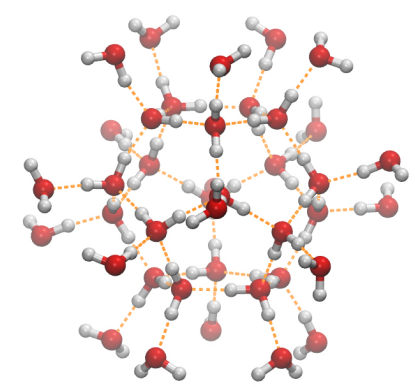

(b)

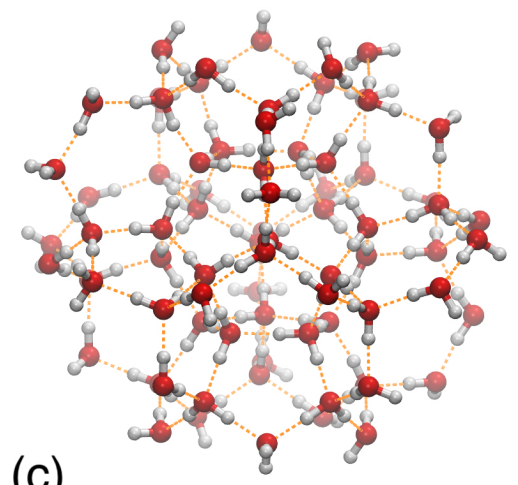

(c)

FIG. 1. Structure of the three sII clathrate hydrate domain (a)-(c) considered in this study. System (a) contains 20 water molecules, system (b) contains 40 water molecules (2 layers) and system (c) contains 76 water molecules (3 layers). 


\section{B. Potential energy surface}

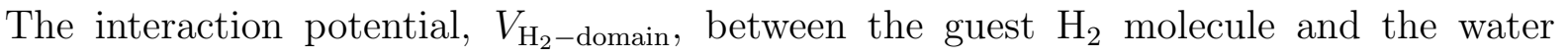
molecules for a given clathrate domain is expressed as:

$$
V_{\mathrm{H}_{2}-\text { domain }}\left(\mathbf{q}_{h}, \boldsymbol{\Xi}\right)=\sum_{w=1}^{n} V_{\mathrm{H}_{2}-\mathrm{H}_{2} \mathrm{O}}\left(\mathbf{q}_{h}, \boldsymbol{\Xi}_{w}\right),+\sum_{w<k} V_{\mathrm{H}_{2} \mathrm{O}-\mathrm{H}_{2} \mathrm{O}}\left(\boldsymbol{\Xi}_{w}, \boldsymbol{\Xi}_{k}\right)
$$

where $\mathbf{q}_{h} \equiv\{x, y, z, \theta, \varphi\}$ are the coordinates of $\mathrm{H}_{2}$ and $\boldsymbol{\Xi} \equiv\left\{\boldsymbol{\Xi}_{1}, \boldsymbol{\Xi}_{2}, \ldots, \boldsymbol{\Xi}_{n}\right\}$ are the cartesian coordinates of the $n$ water molecules in vectorial notation. The term $V_{\mathrm{H}_{2}-\mathrm{H}_{2} \mathrm{O}}$ is the $5 \mathrm{D}$ (rigid rotor approximation) pair interaction between $\mathrm{H}_{2}$ and a $\mathrm{H}_{2} \mathrm{O}$ molecule and the indexes $w$ and $k$ run over the water molecules in the system. We use a simple SPC/E pair-potential model for both interaction between the guest $\mathrm{H}_{2}$ molecule and surrounding

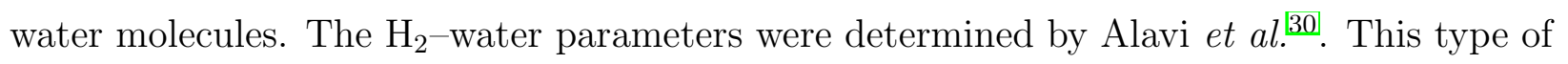
semi-empirical potential has been used in the study of Powers et al..$^{20}$ and shown to account implicitly for many-body effects. This semi-empirical potential can be written simply as a sum of Coulomb and Lennard--Jones (LJ) terms for each type of interaction for all molecules in the system:

$$
V_{\mathrm{H}_{2}-\mathrm{H}_{2} \mathrm{O}}=\sum_{a \in \mathrm{H}_{2}} \sum_{b \in \mathrm{H}_{2} \mathrm{O}} \frac{q_{a} q_{b}}{r_{a b}}+4 \epsilon_{\mathrm{O}-\mathrm{H}_{2}}\left[\left(\frac{\sigma_{\mathrm{O}-\mathrm{H}_{2}}}{r_{\mathrm{O}-\mathrm{H}_{2}}}\right)^{12}-\left(\frac{\sigma_{\mathrm{O}-\mathrm{H}_{2}}}{r_{\mathrm{O}-\mathrm{H}_{2}}}\right)^{6}\right]
$$

The water-water interactions are also described by the SPC/E pair-potential model, parametrised by Berendsen et al. $\stackrel{31}{\text {. }}$.

$$
V_{\mathrm{H}_{2} \mathrm{O}-\mathrm{H}_{2} \mathrm{O}}=\sum_{i} \sum_{j>i}\left\{4 \epsilon\left[\left(\frac{\sigma}{r_{i j}}\right)^{12}-\left(\frac{\sigma}{r_{i j}}\right)^{6}\right]+\sum_{m \in i} \sum_{n \in j} \frac{q_{m} q_{n}}{r_{m n}}\right\}
$$

where $i$ and $j$ runs individually over the atoms of two given water molecules. The parameters we use in this study are reproduced in Table I, for convenience.

Note that in order to accelerate the calculations, we used a well-known approximation for

the computation of $1 / \sqrt{r_{i j}^{2}}$ (InvSqrt, see Ref. 32) which alters slightly the potential energy values compared to a standard calculation of the PES function. We show however (vide infra) that this effect is well below the accuracy of the PES.

\section{Fragment-based rigid-body Quantum Diffusion Monte Carlo}

In this work, we use rigid-body Quantum Diffusion Monte Carlo (RB-DMC) to solve the Schrödinger equation. This approach simulates a diffusion process in imaginary time 
TABLE I. SPC/E parameters used for the $\mathrm{H}_{2}$-water and water-water global potential energy surface. Each water molecule has the same fixed geometry $(d(\mathrm{O}-\mathrm{H})=0.9572 \AA$ and $\mathrm{H}-\mathrm{O}-\mathrm{H}=$ $\left.104.52^{\circ}\right)$. The guest $\mathrm{H}_{2}$ molecule is defined by $d(\mathrm{H}-\mathrm{H})=0.7509 \AA$ and its centre of mass $d\left(\mathrm{H}-\mathrm{H}_{\text {c.m. }}\right)=d(\mathrm{H}-\mathrm{H}) / 2$. We use Lorenz-Bertholet combination rules for each LJ potential term.

\begin{tabular}{lrrr}
\hline \hline Atom type & $q_{i}(\mathrm{e})$ & $\epsilon\left(\mathrm{kcal} . \mathrm{mol}^{-1}\right)$ & $\sigma(\AA)$ \\
\hline $\mathrm{H}_{\text {c.m. }}$ (guest) & -0.9864 & 0.068165 & 3.038 \\
$\mathrm{H}$ (guest) & +0.4932 & 0.000000 & 0.000 \\
$\mathrm{O}$ (water) & -0.8476 & 0.155402 & 3.166 \\
$\mathrm{H}$ (water) & +0.4238 & 0.000000 & 0.000 \\
\hline \hline
\end{tabular}

on a given potential energy surface. The general RB-DMC approach has been described in detail in Ref 33. In brief, we use an energy-shifted time-dependant Schrödinger equation for a system of $N$ particules and perform a Wick rotation which transforms real time $t$ into imaginary time $\tau=i t$, we obtain :

$$
\hbar \frac{\partial \Psi(\vec{r}, \tau)}{\partial \tau}=\sum_{j=1}^{N} \frac{\hbar^{2}}{2 m_{j}} \Psi(\vec{r}, \tau)-\left[V(\vec{r})-E_{r e f}\right] \Psi(\vec{r}, \tau)
$$

where $E_{r e f}$ is the energy-shift term.

This transformation allows the problem to be re-cast as a propagation problem, with the knowledge that $\lim _{\tau \rightarrow+\infty} \Psi(\vec{r}, \tau)=\phi_{0}(\vec{r})$. We use a rigid-body formulation of the Green's function to propagate step by step an initial wave function $\Psi(\vec{r}, 0)$ in imaginary time :

$$
\Psi(\vec{r}, \tau+\Delta \tau)=\int G\left(\vec{r} \rightarrow \vec{r}^{\prime}, \Delta \tau\right) \Psi\left(\vec{r}^{\prime}, \tau\right) d^{3} \vec{r}
$$

with the following separable short time approximation of the Green's function:

$$
\begin{aligned}
G\left(\vec{r} \rightarrow \vec{r}^{\prime}, \Delta \tau\right)= & \prod_{i}\left\{\left(\frac{\mathcal{M}_{i}}{h \Delta \tau}\right)^{3 / 2} \exp \left[\frac{-\mathcal{M}_{i}(\delta x)_{i}^{2}}{2 \hbar \Delta \tau}\right]\right\} \\
& \times \prod_{i}\left\{\prod_{j=1}^{3}\left(\frac{I_{i, j}}{h \Delta \tau}\right)^{3 / 2} \exp \left[\frac{-I_{i, j}\left(\delta \theta_{j}\right)_{i}^{2}}{2 \hbar \Delta \tau}\right]\right\} \\
& \times \exp \left[-\frac{\left(V(\vec{r})-E_{r e f}\right) \Delta \tau}{\hbar}\right]
\end{aligned}
$$

Where $\mathcal{M}_{i}$ corresponds to the total mass and $I_{i, j}$ corresponds to the $j$-th moment of inertia of molecule $i$. We use $(\delta x)_{i}$ and $\left(\delta \theta_{j}\right)_{i}$ to indicate infinitesimal displacements of the centre 
of mass and angular orientation of molecule $i$, respectively. Note that in this expression, we have split the kinetic terms of the propagator into a translational part and a rotational part, similarly to what is described in Ref. 34, and use a quaternion representation of the angular displacements (see Ref. 33).

In order to allow a flexible description of the quantum properties of each system, we have implemented a fragment approach to give us the ability to switch kinetic parts of the Green's function on or off for each molecule. Our fragment-based approach enables us, for example, to propagate a $\mathrm{H}_{2}$ guest molecule using its full translational and rotational Green's function while keeping a fixed clathrate cage, or inside a cage of rotating water molecules each with a fixed centre of mass. This latter approach enables us to preserve the structure of the clathrate cage, while still including rotational quantum effects.

The simulations were performed using a revised version of the XDMC code developed by Benoit ${ }^{35}$ (see also Ref. 33 for implementation details). We used 2000 replicas for all simulations, with a stabilisation period of 10000 cycles along with $\Delta \tau=30$ a.u.. Each simulation used an averaging phase of $60000 \times 100$ cycles with $\Delta \tau=15$ a.u, except for the translation-rotation simulations for fixed clathrate cages that only required $1500 \times 100$ cycles. These parameters were chosen to ensure an acceptable statistical error in the calculations below $13 \mathrm{~cm}^{-1}$ while maintaining a reasonable computational cost. We performed three types of RB-DMC calculations:

- A calculation of the translation-rotation ground state of $\mathrm{H}_{2}$ in the three fixed clathrate cages in order to validate our approach compared to the Smolyak solver.

- A calculations of the translation-rotation ground state of $\mathrm{H}_{2}$ inside the three clathrate cages where the closest 20 water molecules are allowed to rotate around their fixed centre of mass.

- RB-DMC calculations where the position of $\mathrm{H}_{2}$ is fixed at a series of pre-determined positions determined by the Smolyak solver in order to construct and adiabatic potential energy surface.

We use the descendent-weighting approach to compute the 3-D quantum probability distribution of hydrogen atoms in the system, for those molecules that are not held fixed during the calculation. These are represented as isosurfaces and indicate the extent of proton delocalisation in the system (see also Ref. [36). 


\section{Translation-Rotation Bound States Calculations}

The main limitation of our RB-DMC calculations is that we can only compute the translation-rotation ground state of $\mathrm{H}_{2}$ for a given water domain. To overcome this problem, we use an adiabatic approximation. Within this scheme, we consider that the coupling between the water molecules and the molecular hydrogen $\left(\mathbf{q}_{h} \equiv\{x, y, z, \theta, \varphi\}\right)$ is weak. Consider the matrix element for the water domain containing $n$ water molecules interacting with molecular hydrogen:

$$
\left\langle\Psi\left(\mathbf{q}_{h}, \boldsymbol{\Xi}\right)|\hat{H}| \Psi\left(\mathbf{q}_{h}, \boldsymbol{\Xi}\right)\right\rangle
$$

where $\Psi\left(\mathbf{q}_{h}, \boldsymbol{\Xi}\right)$ is the total wave function, $\hat{H}$ the total Hamiltonian and $\mathbf{q}_{h}$ the five coordinates describing the motion of molecular $\mathrm{H}_{2}$. Because of the weak coupling described above, $\Psi$ may be expressed approximately as the product of a function dependent solely on $\mathbf{q}_{h}$, $\psi_{\alpha}\left(\mathbf{q}_{h}\right)$ and a fonction $\phi\left(\boldsymbol{\Xi} ; \mathbf{q}_{h}\right)$ dependent explicitly on all other coordinates of the system $\boldsymbol{\Xi}$ and parametrically on $\mathbf{q}_{h}$. The Hamiltonian may thus be factorised as:

$$
\hat{H}=\hat{T}_{h}+\hat{H}_{w}
$$

where $\hat{T}_{h}$ is the kinetic energy operator of molecular hydrogen described using $\{x, y, z, \theta, \varphi\}$ and $\hat{H}_{w}$ is the Hamiltonian of the $n$ water molecules interacting with molecular hydrogen. This gives:

$$
\left\langle\psi_{\alpha}\left(\mathbf{q}_{h}\right) \phi\left(\boldsymbol{\Xi} ; \mathbf{q}_{h}\right)\left|\left(\hat{T}_{h}+\hat{H}_{w}\right)\right| \psi_{\alpha}\left(\mathbf{q}_{h}\right) \phi\left(\boldsymbol{\Xi} ; \mathbf{q}_{h}\right)\right\rangle
$$

and through RB-DMC we obtain:

$$
\hat{H}_{w} \phi\left(\boldsymbol{\Xi} ; \mathbf{q}_{h}\right)=\mathcal{E}\left(\mathbf{q}_{h}\right) \phi\left(\boldsymbol{\Xi} ; \mathbf{q}_{h}\right)
$$

giving

$$
\left\langle\psi_{\alpha}\left(\mathbf{q}_{h}\right)\left|\hat{T}_{h}+\mathcal{E}\left(\mathbf{q}_{h}\right)\right| \psi_{\alpha}\left(\mathbf{q}_{h}\right)\right\rangle
$$

Thus the problem is effectively reduced to a 5-dimensional problem. The translation-rotation bound states may then be obtained by diagonalising the Hamiltonian $\hat{H}_{h}$ defined as:

$$
\hat{H}_{h}=-\frac{\hbar^{2}}{2 m}\left(\frac{\partial^{2}}{\partial x^{2}}+\frac{\partial^{2}}{\partial y^{2}}+\frac{\partial^{2}}{\partial z^{2}}\right)+B_{0} \hat{j}^{2}+\mathcal{E}(x, y, z, \theta, \varphi),
$$

where $B_{0}$ is the rotational constant in the vibrational ground state of $\mathrm{H}_{2}, m$ is the mass of molecular hydrogen (twice of the atomic hydrogen mass $1.008 \mathrm{~g} \cdot \mathrm{mol}^{-1}$ ), and the angular 
momentum operator $\hat{j}^{2}$ of the diatomic is expanded in terms of the conjugate momenta of $\theta$ and $\varphi$. The $\mathrm{H}_{2}$ bond length is described in Table $\mathbb{I}$, corresponding to the rotational constants $B_{0}=59.322 \mathrm{~cm}^{-1}$ of the free $\mathrm{H}_{2}$ molecule in its $v=0$ vibrational state.

The Smolyak sparse-grid technique ${ }^{37}$ implemented in ELVIBRot ${ }^{\frac{38}{38}}$ is used to compute the 5D TR eigenstates of the Hamiltonian in Eq. (5). This method avoids the need for constructing a direct-product basis and grid, and has been recently investigated by Avila and Carrington $\left[\frac{[19}{41}\right.$ for the calculation of vibrational energy levels of semi-rigid molecules. Later, the same approach was also proposed by Lauvergnat and Nauts 4244 and has been used to compute the torsional levels of methanol in full dimensionality $(12 \mathrm{D})^{43}$. Furthermore recently, this technique has been used to calculate the vibrational shift of $\mathrm{H}_{2}$ in a clathrate hydrate ${ }^{25}$. In the Smolyak approach, the single large direct-product basis or grid is substituted by a sum of small direct-products, $S_{L_{S}}^{r e p}$ :

$$
S_{L_{S}}^{r e p}=\sum_{L=\left[\ell_{1}, \ldots, \ell_{n}\right]}^{L_{S}-n+1 \leq|L| \leq L_{S}}(-1)^{L_{S}-|L|} C_{n-1}^{L_{S}-|L|} S_{\ell_{1}}^{1} \otimes \ldots S_{\ell_{n}}^{n}
$$

In the expression above, $S_{\ell_{i}}^{i}$ represents the $i^{\text {th }}$ primitive basis or grid. The parameter $\ell_{i}$ defines the size of this primitive basis, $n b_{i}\left(\ell_{i}\right)$, or grid, $n q_{i}\left(\ell_{i}\right)$ (see the table below). Therefore, for a given, $i$, several basis sets and grids are required to build this non-direct product grid or basis. Its size is determined through the parameter $L_{S}$ and the range of the sum, $L_{S}-n+1 \leq|L| \leq L_{S}$ where $|L|=\sum \ell_{i}$.

For this system, four $(n=4)$ types of primitive basis are required: 3 harmonic oscillator basis sets and the spherical harmonics to describe, respectively, the translation of $\mathrm{H}_{2}$ (associated to the $x, y, z$ coordinates) and the rotation of $\mathrm{H}_{2}$ (coordinates $\theta$ and $\varphi$ ). The corresponding primitive grids are, respectively, the Gauss Hermite quadrature and the Lebedev grid points. For the harmonic oscillator basis sets, the size of this primitive basis or grids is given by a simple expression, $n b_{i}\left(\ell_{i}\right)=n q_{i}\left(\ell_{i}\right)=1+2 \cdot \ell_{i}(i=1,2,3)$. However, for the spherical harmonic basis, the value $j_{\max }$ is defined initially with $\ell_{4}\left(j_{\max }=\ell_{4}\right)$, then the size of basis set, $n b_{4}$, is the usual expression, $\left(j_{\max }+1\right)^{2}$. The size of the Lebedev grid, $n q_{4}$, is chosen to integrate exactly the overlap matrix.

In the present study, a RB-DMC calculation has to be performed for each grid point to obtain an adiabatic 5D-potential, $\mathcal{E}(x, y, z, \theta, \varphi)$ and, since each RB-DMC calculation take 
TABLE II. Numbers of primitive basis functions, $n b_{i}$ and grid points $n q_{i}$ as function of the parameter $\ell_{i}$.

\begin{tabular}{cccccccc}
\hline \hline$\ell_{i}$ & 0 & 1 & 2 & 3 & 4 & 5 & 6 \\
\hline \multicolumn{7}{c}{ Translation, HO: $i=1,2,3$} \\
$n b_{i}$ and $n q_{i}$ & 1 & 3 & 5 & 7 & 9 & 11 & 13 \\
\hline \multicolumn{7}{c}{ Rotation, $Y_{j}^{m}$} & $\left(0 \leqslant j \leqslant j_{\text {max }}\right): i=4$ \\
$j_{\text {max }}$ & 0 & 1 & 2 & 3 & 4 & 5 & 6 \\
$n b_{4}$ & 1 & 4 & 9 & 16 & 25 & 36 & 49 \\
$n q_{4}$ (Lebedev) & 6 & 6 & 14 & 26 & 38 & 50 & 74 \\
\hline \hline
\end{tabular}

about 58 hours on a 4-cores computer, we need to minimise the total number of grid points. We note that $L_{S}=6$ is sufficient to converge our results to within $0.1 \mathrm{~cm}^{-1}$ for the first seven levels on a rigid cage model with 76 water molecules (this corresponds to 2471 basis functions and 41222 grids points), but with $L_{S}=3$ (180 basis functions and 1618 grids points), the largest error is $1.6 \mathrm{~cm}^{-1}$ only. This accuracy is acceptable to use this grid to compute the RB-DMC adiabatic potential. Furthermore to minimise the RB-DMC computational time, the SPC/E potential was slightly modified (see section IIB). The largest difference of the first seven levels between two calculations with and without this modification using the rigid cage is smaller than $0.5 \mathrm{~cm}^{-1}$ and therefore perfectly acceptable. Finally, with the primitive grids used (Gauss Hermite and Lebedev), a given point can be present several times in the sum of direct-product grids. Therefore, the RB-DMC computations were run only for the 349 unique grid points $\left(L_{S}=3\right)$.

One great advantage of the Smolyak sparse-grid approach is the reduction of the number of grid points with respect to a full direct-product grid. For the present study, the nondirect-product basis set (with $L_{S}=3$ ) is 30 times smaller than the direct-product one (5488 basis functions) and the Smolyak grid is 5.5 times smaller than the direct-product one (8918 grid points).

The subsequent quantum calculations are relatively standard and the wave functions are expanded on a basis set and the corresponding Hamiltonian is diagonalised directly. 


\section{RESULTS AND DISCUSSIONS}

In order to validate our fragment-based RB-DMC, we first calculate the translationrotation ground state of the $\mathrm{H}_{2}$ guest molecule in each fixed water cage. Our results are shown in the upper section of Table [II, along with a reference calculation using the Smolyak approach $\left(L_{S}=4\right)$. We observe that for each cage, the difference between our reference and our RB-DMC calculations (approx. $0.4 \mathrm{~cm}^{-1}$ ) are of the same magnitude as the uncertainty of the Monte-Carlo simulations $\left(0.3 \mathrm{~cm}^{-1}\right)$. This further confirms the reliability of RB-DMC for these systems, as was also mentioned in Ref. 25.

We use each energy minimum and the energy of the corresponding RB-DMC translationrotation ground state to compute the zero-point energy ( $\mathrm{ZPE}$ ) of $\mathrm{H}_{2}$ in the three frozen cage. We note that the ZPE is similar (around $120 \mathrm{~cm}^{-1}$ ) for the three cages, with the smallest cage, $n=20$, having the largest ZPE, while the larger cages have a similar ZPE.

Next, we examine the effect of including the rotational degrees of freedom for the water molecules nearest to the guest molecule (first shell, 20 water molecules). However, to preserve the structure of the clathrate cages, we keep the centre of masses of the water molecules fixed. Our results are shown in the lower section of Table [II]. In order to compute the translation-rotation $\mathrm{ZPE}$ of $\mathrm{H}_{2}$ with the added degrees of freedom, we perform two RBDMC calculations: one where the guest molecule is allowed full translational and rotational degrees of freedom and the water molecules are allowed to rotate; and another calculation where only the water molecules are allowed to rotate while the guest molecule is kept fixed at its minimum energy position. Here we notice that there is a marked difference between the ZPE computed for the smaller cage $(n=20)$ and the values obtained for the two larger cages. The ZPE is larger for $n=20\left(394 \pm 13 \mathrm{~cm}^{-1}\right)$, possibly due to the more delocalised nature of this small cage model. Indeed, both larger models include a second solvation

shell that confers more rigidity to the system. This is also evident from the probability distribution (hydrogen density) isosurfaces discussed below.

The RB-DMC calculations enable us to compute the probability distribution of atoms and therefore the quantum delocalisation of the nuclear wave function. In Fig. 2, the 3-D isosurface of the hydrogen atoms is shown as an almost spherical surface. If the cages are held rigid (the three left panels, A, C, E in Fig. 2), the size of the hydrogen 3-D isosurfaces are almost identical. The same holds true when the water rotational degrees of freedom 

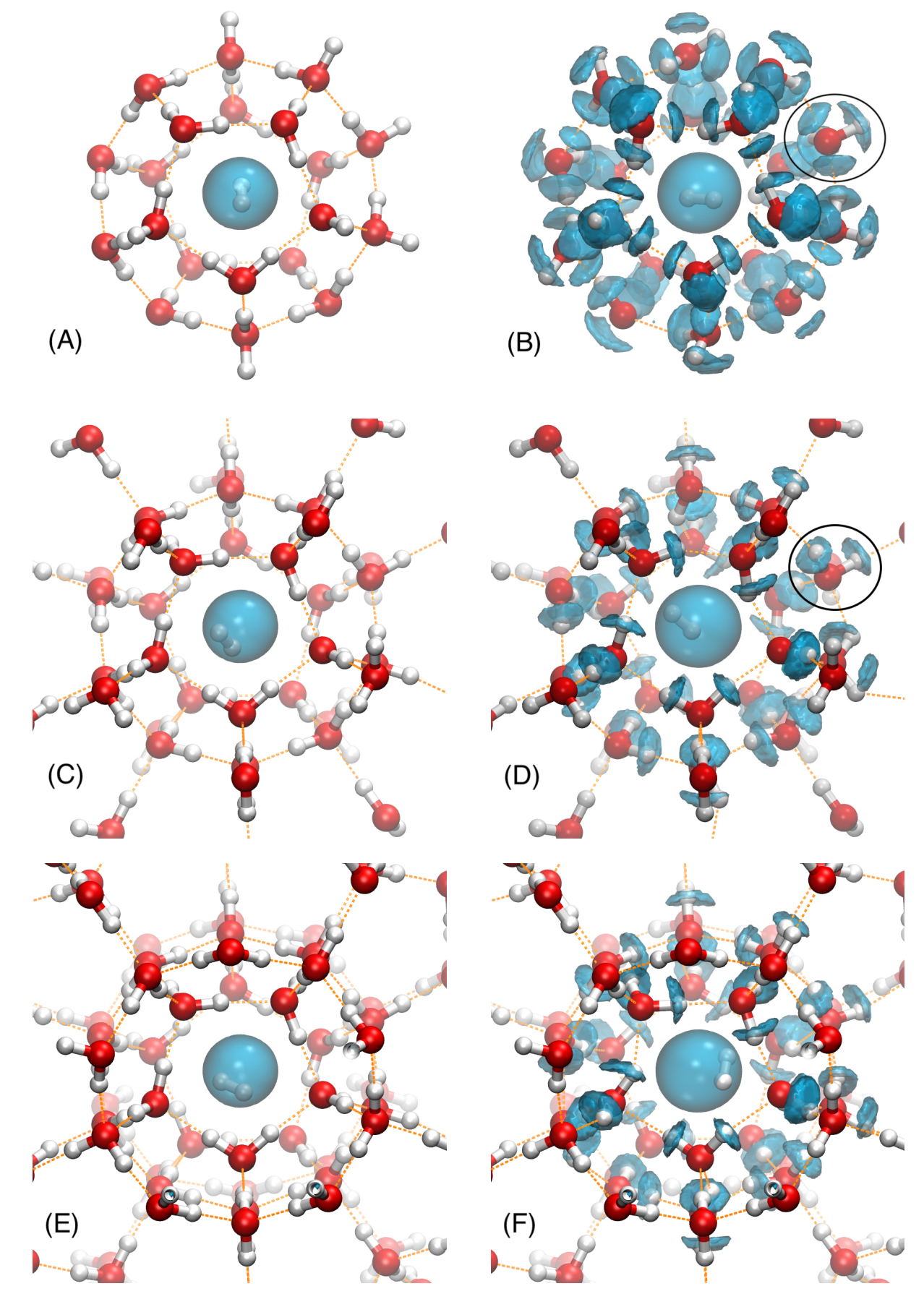

FIG. 2. 3-D isosurfaces of the hydrogen probability distribution (H-density) obtained from RBDMC calculations for $\mathrm{H}_{2}$ in three types of $\left(\mathrm{H}_{2} \mathrm{O}\right)_{n}$ clathrate cages. Panel (A) shows the TR ground state $\mathrm{H}$-density for $\mathrm{H}_{2}$ in a fixed $n=20$ cage, while Panel (B) shows the H-density when the cage molecules are allowed to rotate. Panel (C) and (D) show the same two situations but for a larger $n=40$ cage. Finally, Panel (E) and (F) shows the same two situations for the largest cage considered in this study $(n=76)$. All isosurfaces display a level of $0.05 \mathrm{H}$-density/ $\AA^{3}$. 
TABLE III. Computed energies of the translation-rotation ground state of $\mathrm{H}_{2}$ in three different clathrate cages $\left(\mathrm{H}_{2} \mathrm{O}\right)_{n}, n=20,40,76$ obtained using the Smolyak approach $\left(L_{S}=4\right)$ and the RB-DMC technique. The uncertainty of the RB-DMC results is $\pm 0.3 \mathrm{~cm}^{-1}$ for the fixed caged calculations and $\pm 13 \mathrm{~cm}^{-1}$ for the calculations including the rotational degrees of freedom for the water molecules.

\begin{tabular}{|c|c|c|c|}
\hline & \multicolumn{3}{|c|}{$n$} \\
\hline & 20 & 40 & 76 \\
\hline \multirow[t]{2}{*}{ Minimum energy } & -77069.2 & -136228.5 & -304949.2 \\
\hline & \multicolumn{3}{|c|}{ Rigid cage calculations } \\
\hline$E_{\text {Smolyak }}^{0}$ & -76932.2 & -136108.5 & -304824.5 \\
\hline$E_{\mathrm{RB}-\mathrm{DMC}}^{0}$ & -76932.5 & -136109.0 & -304824.9 \\
\hline$E_{\mathrm{RB}-\mathrm{DMC}}^{0}-E_{\text {Smolyak }}^{0}$ & 0.3 & 0.5 & 0.4 \\
\hline \multirow[t]{2}{*}{$\mathrm{ZPE}_{\mathrm{RB}-\mathrm{DMC}}$} & 136.7 & 119.5 & 124.3 \\
\hline & \multicolumn{3}{|c|}{ Rotating water molecules calculations } \\
\hline$E_{\mathrm{RB}-\mathrm{DMC}}^{0}$ & -64529 & -114806 & -282779 \\
\hline$E_{\mathrm{RB}-\mathrm{DMC}}^{0}\left(\right.$ fixed $\left.\mathrm{H}_{2}\right)$ & -64923 & -115060 & -283042 \\
\hline $\mathrm{ZPE}_{\mathrm{RB}-\mathrm{DMC}}$ & 394 & 254 & 263 \\
\hline
\end{tabular}

are included (right panels, B, D, F in Fig. 2). However, for given a cage (for $n=20$, for example, panels $\mathrm{A}$ and $\mathrm{B}$ ), the hydrogen 3-D isosurfaces appears larger for the cage with rotating water molecules than for the rigid counterpart. Indeed, when the water molecules are not held fixed in the cage, they can easily twist out of their equilibrium positions (see isosurfaces in the right panels). The delocalisation of the water hydrogen atoms that would otherwise point inside the cage in their fixed configuration leaves more space available for the $\mathrm{H}_{2}$ motion and thus create a slightly larger cavity for the guest.

The 3-D isosurfaces of the rotating water molecules (see Fig. 2) show a markedly different behaviour when comparing the small cage with $n=20$ water molecules (panel B) to the two larger ones containing a $2^{\text {nd }}$ (panel D) or a $2^{\text {nd }}$ and $3^{\text {rd }}$ shells (panel F). Indeed, for the small cage, the delocalisation of the hydrogen atom of the water molecules is more pronounced 
than for the two other cages. This feature can be understood as follows:

(i) The highlighted water molecule in the small cage (Fig. 2B) is delocalised over four probability distribution basins showing at least two different local conformations. This implies that there are a number of possible hydrogen-bond networks present in this delocalised RB-DMC ground state. Such dynamical behaviour is likely due to the absence of a rigid $2^{\text {nd }}$ or $3^{\text {rd }}$ water shells and it can be viewed as a $\mathrm{H}_{2}$ molecule inside of a cluster of 20 water molecules rather than inside a realistic water clathrate model.

(ii) The highlighted water molecule of the medium-size cage (Fig. 2D) is only delocalised over two probability distribution basins. The hydrogen atoms of the water molecules are still delocalised but are constrained vibrate around their equilibrium positions due to the presence of the $2^{\text {nd }}$ or $3^{\text {rd }}$ water shells.

In the present study, we want address the role of quantum delocalisation of waterframework protons from an explicit quantum point of view and therefore at $T=0 \mathrm{~K}$. The results in table IV show the quantum effects caused by the twisting water motions of the clathrate hydrate, obtained adiabatically with RB-DMC calculations, coupled to a 5D-quantum treatment of $\mathrm{H}_{2}$. The calculated energy levels obtained with a rigid cage with $n=40$ water molecules and those obtained while allowing 20 of the 40 water molecules to rotate are presented in table IV] (columns 3 and 4). The energy differences between these two calculations are shown in column 5. Those results show that the rotational quantum effect of the water molecules perturbs noticeably the energy levels:

(i) for the translational levels (at $j=0$ ), the all energies obtained with the quantum traitement of the rotating water molecules (column 4) increase with respect to the ones obtained with the rigid cage (column 3). This is particularly noticeable for the second translational component. Furthermore, the energy splitting difference between the third and the first translational components (fifth line of the table IV) increases slightly as well. However, this effect is small and probably not relevant given the accuracy of our calculation $\left(1-2 \mathrm{~cm}^{-1}\right)$. It is worth noting that Powers et al ${ }^{[20}$ show similar results (no translation splitting increase) for the translation motion of $\mathrm{H}_{2}$ but with $j=1$.

(ii) for the rotational levels at $j=1$, the energies of the first and the second components 
TABLE IV. Comparison between experimental (from Ref. 45, column 2) fundamental translational transitions and the rotational $j=0 \rightarrow 1$ transitions of $\mathrm{H}_{2}$ in sII clathrate hydrate and the calculated ones with a cage with 76 water molecules (column 3, rigid cage and column 4, cage with rotating water molecules). The difference between the two calculated transitions are presented in the $5^{\text {th }}$ column. All transitions in $\mathrm{cm}^{-1}$. For the translational and rotational $(j=0 \rightarrow 1)$ transitions, the splitting is defined as the energy difference between the $3^{\text {rd }}$ ans the $1^{\text {st }}$ components.

\begin{tabular}{ccccc}
\hline \hline & Exp. & Rigid & Rotating & $\Delta_{\text {Rotating-Rigid }}$ \\
\hline \multicolumn{5}{c}{ Translation } \\
I & $\mathbf{7 1 . 0}$ & 78.5 & 80.5 & +2.0 \\
II & $\mathbf{8 0 . 2}$ & 78.7 & 85.1 & +6.4 \\
III & $\mathbf{1 0 1 . 1}$ & 102.9 & 107.7 & +4.8 \\
Splittings & $\mathbf{3 0 . 1}$ & 24.4 & 27.2 & +2.8 \\
\hline & & Rotation, $j=1$ & \\
I & $\mathbf{1 1 0 . 0}$ & 110.5 & 108.6 & -1.9 \\
II & $\mathbf{1 1 6 . 5}$ & 118.5 & 117.8 & -0.7 \\
III & $\mathbf{1 2 2 . 1}$ & 120.9 & 124.1 & +3.2 \\
Splittings & $\mathbf{1 2 . 1}$ & 10.4 & 15.5 & +5.1 \\
\hline \hline
\end{tabular}

(column 4) decrease slightly with respect to the column 3 (rigid cage). For the the third component, the increase compared to the rigid value is more pronounced. However, the main feature is the rotational splitting $\left(15.5 \mathrm{~cm}^{-1}\right)$, which increases noticeably compared to the value obtained for the rigid cage $\left(10.4 \mathrm{~cm}^{-1}\right)$. Powers et al ${ }^{20}$ also noticed this effect with their classical molecular simulation of the water motions and therefore at finite temperature, while in the present work, we show that this effect is still important at $T=0 \mathrm{~K}$. 


\section{CONCLUSIONS}

We have performed 5D calculations of the TR levels of $\mathrm{H}_{2}$ encapsulated in small dodecahedral clathrate hydrates, taking into account the quantum delocalisation of the protons nuclei in the water framework. Our calculations were performed using an adiabatic decoupling scheme between the slow hindered rotational motions of the first-shell water molecules and the fast translation-rotation motions of the guest molecular hydrogen. The relatively slow motion of the water molecules is solved using a RB-DMC algorithm to compute an effective adiabatic potential for the guest $\mathrm{H}_{2}$ molecule in a quantum clathrate environment. A Smolyak sparse-grid scheme was applied to determine the 5D dimensional TR bound states on this adiabatic potential. The condensed phase environment was explicitly considered by adding two successive layers of water molecules with fixed geometries to the first shell composed of rotating water molecules (20 molecules). The surrounding second and third water shells are thus used to constrain and limit the rotational motion of the first shell water molecules, leading to a more realistic model for condensed phase conditions.

Our results highlight the role of quantum delocalisation effect of protons nuclei in the water framework even at $T=0 \mathrm{~K}$. This work represents the first study of the quantum effect of the nanoscale environment on a guest molecule from a spectroscopic perspective. The computed TR levels shows that the splitting of the translational fundamental is not sensitive to the inclusion of the quantum delocalisation of its surrounding. Conversely, the $j=1$ rotational splitting is increased by the inclusion of the rotational motions of water molecules, as observed by Bačić and co-workers ${ }^{20}$ using a static approach. Arguably, the averaging of

classical structures does not provide a direct evaluation of the quantum dynamical effect of the host system. However, our observed agreement between static and quantum dynamical approach seems to indicate that the averaged description considered in Ref. ${ }^{20}$ was able to capture the main effect of proton delocalisation in the water framework.

Finally, in this study, we only consider the adiabatic quantum treatment of the rotational degrees of freedom of the water molecules around a fixed position. Yet our new approach could also explore the inclusion of translational degrees of freedom for the cage, which would constitute a further step towards a full quantum treatment of clathrate hydrates and their guest molecules. 


\section{CONFLICTS OF INTEREST}

There are no conflicts of interest to declare.

\section{ACKNOWLEDGEMENTS}

D.M.B. acknowledges the Viper High Performance Computing facility of the University of Hull and its support team.

\section{REFERENCES}

${ }^{1}$ V. V. Struzhkin, B. Militzer, W. L. Mao, H. K. Mao, and R. J. Hemley, Chem. Rev. 107, $4133(2007)$.

${ }^{2}$ W. L. Mao, C. A. Koh, and E. D. Sloan, Phys. Today 60, 42 (issue 10, 2007).

${ }^{3}$ W. L. Mao and H. K. Mao, Proc. Natl. Acad. Sci. U.S.A. 101, 708 (2004).

${ }^{4}$ F. Schüth, Nature 434, 712 (2005).

${ }^{5}$ Y. A. Dyadin, E. G. Larionov, A. Y. Manakov, F. V. Zhurko, E. Y. Aladko, T. V. Mikina, and V. Y. Komarov, Mendeleev Commun. 9, 209 (1999).

${ }^{6}$ W. L. Mao, H. K. Mao, A. F. Goncharov, V. V. Struzhkin, Q. Guo, J. Hu, J. Shu, R. J. Hemley, M. Somayazulu, and Y. Zhao, Science 297, 2247 (2002).

${ }^{7}$ K. A. Lokshin, Y. Zhao, D. He, W. L. Mao, H. K. Mao, R. J. Hemley, M. V. Lobanov, and M. Greenblatt, Phys. Rev. Lett. 93, 125503 (2004).

${ }^{8}$ M. Xu, Y. Elmatad, F. Sebastianelli, J. W. Moskowitz, and Z. Bačić, J. Phys. Chem. B 110, 24806 (2006).

${ }^{9}$ F. Sebastianelli, M. Xu, Y. Elmatad, J. W. Moskowitz, and Z. Bačić, J. Phys. Chem. C 111, 2497 (2007).

${ }^{10}$ F. Sebastianelli, M. Xu, D. K. Kanan, and Z. Bačić, J. Phys. Chem. A 111, 6115 (2007).

${ }^{11}$ M. Xu, F. Sebastianelli, and Z. Bačić, J. Phys. Chem. A 111, 12763 (2007).

${ }^{12}$ M. Xu, F. Sebastianelli, and Z. Bačić, J. Chem. Phys. 128, 244715 (2008).

${ }^{13}$ M. Xu, F. Sebastianelli, Z. Bačić, R. Lawler, and N. J. Turro, J. Chem. Phys. 129, 064313 (2008).

${ }^{14}$ F. Sebastianelli, M. Xu, and Z. Bačić, J. Chem. Phys. 129, 244706 (2008).

${ }^{15}$ M. Xu, F. Sebastianelli, and Z. Bačić, J. Phys. Chem. A 113, 7601 (2009). 
${ }^{16}$ A. Witt, F. Sebastianelli, M. E. Tuckerman, and Z. Bačić, J. Phys. Chem. C 114, 20775 (2010).

${ }^{17}$ M. Xu and Z. Bačić, Phys. Rev. B 84, 195445 (2011).

${ }^{18}$ M. Celli, A. Powers, D. Colognesi, M. Xu, Z. Bačić, and L. Ulivi, J. Chem. Phys. 139, 164507 (2013).

${ }^{19}$ D. Colognesi, A. Powers, M. Celli, M. Xu, Z. Bačić, and L. Ulivi, J. Chem. Phys. 141, 134501 (2014).

${ }^{20}$ A. Powers, O. Marsalek, M. Xu, L. Ulivi, D. Colognesi, M. E. Tuckerman, and Z. Bačić, J. Phys. Chem. Lett. 7, 308 (2016).

${ }^{21}$ P. M. Felker and Z. Bačić, J. Chem. Phys. 144, 201101 (2016).

${ }^{22}$ P. M. Felker and Z. Bačić, J. Chem. Phys. 145, 084310 (2016).

${ }^{23}$ P. M. Felker and Z. Bačić, J. Chem. Phys. 146, 084303 (2017).

${ }^{24}$ P. Valiron, M. Wernli, A. Faure, L. Wiesenfeld, C. Rist, S. Kedzuch, and J. Noga, J. Chem. Phys. 129, 134306 (2008).

${ }^{25}$ A. Powers, Y. Scribano, D. Lauvergnat, E. Mebe, D. M. Benoit, and Z. Bačić, J. Chem. Phys. 148, 144304 (2018).

${ }^{26}$ A. Valdeś and G. J. Kroes, J. Phys. Chem. C 116, 21664 (2012).

${ }^{27}$ P. M. Felker, J. Chem. Phys. 141, 184305 (2014).

${ }^{28}$ P. M. Felker, J. Chem. Phys. 138, 174306 (2013).

${ }^{29}$ N. Plattner and M. Meuwly, J. Chem. Phys. 140, 024311 (2014).

${ }^{30}$ S. Alavi, J. A. Ripmeester, and D. D. Klug, J. Chem. Phys. 123, 024507 (2005).

${ }^{31}$ H. J. C. Berendsen, J. R. Grigera, and T. P. Straatsma, J. Phys. Chem. 91, 6269 (1987).

${ }^{32}$ M. Robertson, A Brief History of InvSqrt (BSc. thesis, The University of New Brunswick, 2012).

${ }^{33}$ D. M. Benoit and D. C. Clary, J. Chem. Phys. 113, 5193 (2000).

${ }^{34}$ V. Buch, J. Chem. Phys. 97, 726 (1992).

${ }^{35}$ D. M. Benoit, Xdmc, Quantum Diffusion Monte Carlo code (1999), .

${ }^{36}$ D. C. Clary, D. M. Benoit, and T. van Mourik, Acc. Chem. Res. 33, 441 (2000).

${ }^{37}$ S. A. Smolyak, Soviet Mathematics Doklady 4, 240 (1963).

${ }^{38}$ D. Lauvergnat, ElVibRot: quantum dynamics code, http://pagesperso.lcp.u-psud. fr/lauvergnat/ElVibRot/ElVibRot.html.

${ }^{39}$ G. Avila and T. Carrington, J. Chem. Phys. 131, 174103 (2009). 
${ }^{40}$ G. Avila and T. Carrington, J. Chem. Phys. 134, 054126 (2011).

${ }^{41}$ G. Avila and T. Carrington, J. Chem. Phys. 135, 064101 (2011).

${ }^{42}$ D. Lauvergnat and A. Nauts, Phys. Chem. Chem. Phys. 12, 8405 (2010).

${ }^{43}$ D. Lauvergnat and A. Nauts, Spectrochim. Acta. Part A. 119, 18 (2014).

${ }^{44}$ J. Sarka, D. Lauvergnat, V. Brites, A. G. Császár, and C. Léonard, Phys. Chem. Chem. Phys. 18, 17678 (2016).

${ }^{45}$ L. Ulivi, M. Celli, A. Giannasi, A. J. Ramirez-Cuesta, D. J. Bull, and M. Zoppi, Phys. Rev. B 76, 161401(R) (2007). 